\title{
A SURVEY ON MAC PROTOCOLS FOR AD HOC NETWORKS WITH DIRECTIONAL ANTENNAS
}

\author{
Robert Vilzmann \\ Technische Universität München, Institute of Communication Networks \\ Munich, Germany
}

Christian Bettstetter

DoCoMo Euro-Labs, Future Networking Lab

Munich, Germany

Abstract The application of directional antennas in the mobile devices of wireless ad hoc networks has the potential to increase the network connectivity and capacity. However, new solutions for medium access control (MAC) are needed. This paper provides a survey and overview of recently proposed MAC protocols for this scenario. We summarize problems specific to this setup and categorize proposed protocols.

Keywords: Ad hoc networking, medium access, directional antennas, beamforming

\section{Introduction and Motivation}

D HOC networking promises to provide the technical concepts for a va-
riety of future wireless systems and services, such as short-range data
transfer, vehicular communication, robust emergency services, and multihop
access to infrastructure. An ad hoc network connects mobile devices, referred
to as nodes, which act as relays to provide a communication between remote
nodes in a multi-hop fashion.
Recently, interest in the application of directional (or beamforming) anten-
nas in ad hoc networks has arisen [Ramanathan, 2001]. Due to high antenna
gains, directional antennas are expected to provide increased coverage and con-
nectivity among nodes. Limiting the area in which the transmission signal is
radiated can reduce high-frequency emissions and prolong the battery lifetime.
Furthermore, directional antennas can increase the network capacity by allow-
ing for higher spatial reuse of radio resources. The latter advantage is particu- 
larly alluring since, for omnidirectional antennas, it has been shown in [Gupta and Kumar, 2000] that, under certain models defining successful transmissions, the capacity per node diminishes to zero with an increasing node density. These benefits from the networking layer - in conjunction with increased data rates achieved on the physical layer - may facilitate the provision of quality of service (QoS) in wireless ad hoc networks. For instance, increased transmission ranges may reduce the hop distance between two nodes, and thus reduce the end-to-end delay.

A challenging task in ad hoc networks is the problem of medium access control (MAC). Since sharing the medium has to be controlled in a distributed fashion, centralized MAC approaches, which may easily manage guaranteed (scheduled) access in a typically time slotted manner, are not feasible. Mastering data streams with imposed QoS constraints is thus tough. Moreover, the wireless environment imposes restrictions and impairments that complicate the design of appropriate MAC protocols as compared to fixed (wireline) networks, most notably the half-duplex operation of transceivers, the timevarying, unpredictable and unreliable wireless channel, frequent and bursty channel errors, and location dependent carrier sensing [Gummalla and Limb, 2000]. The problem of location dependent carrier sensing inhibits collision detection mechanisms, at least if only one transceiver unit per node is available.

All above-mentioned MAC issues in wireless networks also apply to the case of directional antennas. Interestingly, when trying to exploit the benefits of directional antennas, additional challenges in the design of MAC protocols arise, especially in mobile ad hoc networks where nodes may move and rotate.

This paper provides a survey and overview of recently proposed MAC protocols for this scenario. We summarize problems specific to this setup and categorize proposed protocols that deal with these problems. The paper is organized as follows. First, Section 2 expands on MAC issues with directional antennas. Next, Section 3 surveys a considerable number of MAC protocols addressing these issues. Section 4 gives a summary and comparison of the proposed approaches. Finally, Section 5 concludes.

\section{Medium Access Problems using Directional Antennas}

The capabilities of directional antennas are typically not fully exploited when using conventional MAC protocols, such as IEEE 802.11. In fact, the network performance may even deteriorate due to issues specific to directional antennas. In the following sections, we summarize the major problems.

\subsection{Neighbor location and main lobe direction}

With omnidirectional antennas, the angular direction of a neighbor (a node that can be reached via a direct link) is not relevant. In the case of directional 
antennas, however, the relative angular position of the neighbors is indeed critical. First, when using beamforming at the transmitter, the node must know where the main lobe of the antenna gain pattern should be directed to maximize the link quality. Second, when increasing the spatial reuse by allowing for simultaneous transmissions in the vicinity of a receiver, it is important to know whether beamforming ensures that no or limited interference is caused at other neighbors.

At least two basic methods for determining the angular position of neighbors exist. The first possibility is to use GPS information. This requires a GPS unit at each node and the exchange of GPS information for the computation of neighbor directions. The dependence on an auxiliary positioning system is generally precarious, and GPS usually does not work indoors. The second possibility is direction-of-arrival (DOA) estimation in the receive path. Besides the complexity of the algorithm and the accuracy of the results, the time to estimate the direction of incoming signals is an important aspect. A comprehensive review of DOA estimation methods can be found in [Godara, 1997]. It is important to note that the topographical position of a node may not be the optimal beamforming direction. This is due to the real signal propagation path, where reflection, diffraction and scattering may occur. Consequently, this is a strong argument for preferring DOA estimation over GPS-based approaches.

In summary, the problem of neighbor discovery is augmented with location or angle of arrival discovery when using. Many MAC protocols for directional antennas assume known node locations.

\subsection{Extended transmission range}

By exploiting the beamforming capabilities of directional antennas, increased transmission ranges can be achieved. For instance, when using a phased array antenna with isotropic antenna elements, the maximum antenna gain roughly equals the number of antenna elements. The high antenna gain corresponds to longer transmission ranges, helping the network to reduce the number of hops between the source and the sink of a packet and increasing the network connectivity by bridging between otherwise disconnected network clusters.

A question relevant in medium access is whether increased ranges can be exploited, and how links ranging beyond the omnidirectional range can be set up. If two nodes can only communicate if they point their main beam toward each other, they are called DD (Directional-Directional)-neighbors. The MMAC protocol [Choudhury et al., 2002], for example, suggests the setup of DD-links through short-range control packets exchanged via multiple hops.

Besides the problem of facilitating longer links, transmission beyond the reserved transmission floor is a MAC-layer issue caused by high antenna gains. 
Problems occur when directionally transmitted data packets cause interference at nodes outside the area which was reserved by exchanging omnidirectional control messages. A possible solution to this problem is to apply means for power control. In this case however the extended transmission range of beamforming antennas is not exploited.

\subsection{Side lobe pattern}

Multi-element array (MEA) antennas are often considered in research on directional antennas in ad hoc networks. An MEA antenna comprising $N$ antenna elements provides $N$ degrees of freedom for beamforming. When a receiving node points a main lobe toward a transmitter, $N-1$ degrees of freedom remain for maximizing the signal-to-interference (SIR) ratio. This can be done by placing antenna gain nulls in the direction of interferers ("null beam forming" or "null steering"). While the angular direction of the main beam and the nulls may be governed by the MAC-layer, the exact antenna gain pattern cannot be assumed to be known to the MAC layer. When steering the main beam around the node, the entire side lobe pattern may change drastically, and even the maximum gain and beamwidth of the main lobe is not constant.

For the MAC-layer, this raises the problem that the exact amount of interference caused through side lobes at (for transmitting nodes) or by (for receiving nodes) neighboring nodes cannot not be known a priori medium access. For a known main beam direction relative to the array orientation the entire beamforming pattern can, in theory, be calculated for a given antenna array configuration (number of antenna elements, symmetry and spacing), see Fig. 1. However, having this data available and eventually making use of it for medium access may be impossible due to complexity issues.

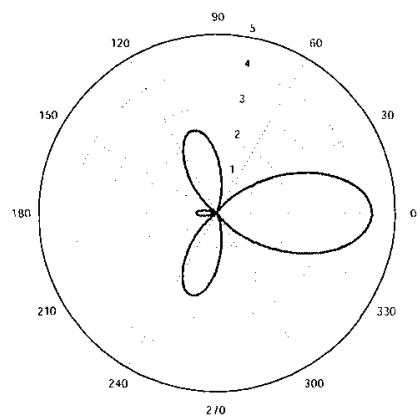

Figure 1. Horizontal section of the antenna gain pattern of a uniform circular array antenna with $N=4$ antenna elements and half-wavelength element spacing, main lobe direction $0^{\circ}$ 
Many proposals for MAC-layer design published recently stick with very simplified antenna models, e.g. simple sector models. Such "areal" models may be very inappropriate.

\subsection{Directional carrier sensing}

In recently proposed MAC protocols for beamforming antennas, directional physical carrier sensing is a frequently used technique that is helpful to some extend. The goal of carrier sensing preceding channel access is to prevent from collisions by signal interference. When a signal is transmitted into a sector in space using a directional antenna, it makes some sense to also limit the carrier sensing to this sector in order to increase the spatial reuse of radio resources.

However, impairments exist: In mobile networks, there is the general problem that sensing the carrier at the transmitter does not accurately reflect the situation at the receiver. This means that the channel may be busy at the intended receiver, while it appears to be free at the transmitter. With directional carrier sensing, there are even more possible scenarios in which a parallel signal transmission may not be detected.

\subsection{New hidden terminals}

For a transmission from Node A to Node B, a Node C is "hidden" with respect to $\mathrm{A}$ if it is located outside the transmission range of Node $\mathrm{A}$, but within the range of Node B. If not blocked, hidden terminals may cause corruptive interference at the receiver. Virtual carrier sensing, such as the request-tosend/clear-to-send (RTS/CTS) exchange in IEEE 802.11, seeks to avoid hidden terminals.

The application of the IEEE 802.11 RTS/CTS scheme to directional antennas can create new kinds of hidden terminals. These new problems arise due to increased directional antenna gains and due to unheard control packets (because of beamforming) [Choudhury et al., 2002].

\subsection{Deafness}

Deafness is another problem that may arise when using beamforming antennas. We would like to give the following definition: A node is deaf with respect to a second node, if it cannot receive from the second node due to its antenna gain pattern, while the node could receive from the second node, if the second node would be in omnidirectional mode. Deafness may thus occur when a receiving node stays beamformed toward its transmitter in between two successive packets (directional carrier sensing), and a third node tries to transmit to the receiving node. According to our above definition, a situation where a currently transmitting node has formed a null gain toward a second node is 
not deafness per se, since the transmitting node could not receive from the second node even if it was in omnidirectional mode (half-duplex operation).

Reference [Choudhury and Vaidya, 2003] contributes with an evaluation of the impact of deafness and addresses the tradeoff between maximizing the benefits of beamforming antennas and the deafness problem. An interesting aspect of the deafness problem is that chains or even deadlock situations are possible where no node can communicate. The authors argue that prolonged deafness can lead to multiple packet drops, which may mislead higher layers. Furthermore, it is emphasized that deafness affects fairness among transmitting nodes.

\subsection{Antenna array rotation and changing beamforming pattern}

In ad hoc networks, MAC decisions have to be made in a distributed manner. Hence, network changes typically entail the need for information exchange, leading to signaling overhead. The performance of MAC protocols thus degrades with increasing system dynamics, and malfunctions may occur in highly dynamic scenarios. Typical sources for network dynamics are time-varying traffic loads and transmission urgencies. Moreover, in wireless systems, the channel can vary rapidly over time. In mobile systems, the (translatory) mobility of all nodes is another source of dynamics.

When using beamforming antennas, antenna array rotations introduce dynamics that used to be unknown as long as ad hoc networks with isotropic antennas were considered. Note that, since the determination of the angular direction of neighbors is typically essential to MAC approaches for directional antennas, the angular direction of neighbors has to be re-determined frequently if mobile nodes are free to rotate.

Even more dynamics is due to the fact that nodes can change their beamforming pattern. In this case, not only the main lobe direction changes. In fact, the entire beamforming pattern (including the side lobes) changes if the main lobe direction of an antenna array is electrically steered. Adaptations of the beamforming pattern may be performed packet-wise. From the protocol and physical point of view, respectively, neighborhood relationships and interference levels may thus completely change over time in the order of one millisecond (assuming a typical packet size of $1 \mathrm{kByte}$ and a transmission rate of $10 \mathrm{Mbit} / \mathrm{s})$.

\section{MAC Protocols for Directional Antennas}

This section discusses MAC protocols designed for controlling medium access if nodes are equipped with directional antennas. Most of these protocols are extensions to the distributed coordination function (DCF) of IEEE 
802.11, which comprises physical and virtual carrier sensing, implemented by an RTS/CTS handshake preceding the data transmission. These protocols are surveyed in Section 3.1. For future ad hoc networks, being either fully selforganizing or an access network for fixed infrastructure networks, it may be necessary to entirely reconceive the MAC problem and develop novel, "non802.11" MAC protocols. Characteristics that are considered to be different from 802.11 comprise in particular scheduled approaches, protocols which do not rely on virtual carrier sensing, and protocols which are not based on the notion of blocking an area by RTS/CTS messages. The rather limited literature on such protocols is discussed in Section 3.2.

\subsection{11-type MAC protocols for directional antennas}

The protocols summarized in the following are based on the IEEE 802.11 DCF protocol. They typically comprise the well-known RTS-CTS-DATAACK procedure, also known as virtual carrier sensing. Another concept known from 802.11 is using a so called Network Allocation Vector (NAV); it keeps track of the time a node has to remain in a blocked state upon overhearing a control message. With a number of directional protocols, the NAV vector is extended for maintaining direction-specific blocking information. It is commonly referred to as Directional NAV (DNAV).

One of the first modifications of DCF that aims at directional antennas was proposed in the Directional MAC (D-MAC) protocol [Ko et al., 2000]. It is a rather straightforward extension of the 802.11 protocol. Here, RTS, DATA, and ACK packets are sent directionally. Alternatively, RTS packets are sent omnidirectionally if none of the directional antennas of the transmitter is blocked. This reduces the probability of control packets collisions.

Another early protocol was the Multi-Hop RTS MAC (MMAC) protocol [Choudhury et al., 2002]. It facilitates DD-links through multi-hop signaling. To this end, a special type of RTS packet is used, the reception of which does not affect DNAV tables.

The Tone-based directional MAC (ToneDMAC) protocol [Choudhury and Vaidya, 2003] seeks to indicate deafness to blocked transmitters and thus increase fairness. Using omnidirectional tones after the DATA/ACK exchange, both transmitter and receiver indicate that they were recently engaged in communication. Thus, a neighboring node can realize deafness if it overhears a tone from its intended receiver. In this case, the node reduces its contention window to the minimum value and has thus a fair chance to win the next channel contention. Multiple tones are used, and a node has to be able to identify the transmitter of a tone. It is assumed that a set of tone frequencies and durations is available. Further, the transmitter of a tone can be determined with a certain probability by means of its unique identifier and a hash function. The 
authors propose to exploit location information about neighboring nodes in order to reduce the probability of tone mismatching.

In [Nasipuri et al., 2000], Nasipuri et al. focus on interference reduction by directional transmission of data packets. This protocol requires all nodes to maintain the antenna orientation at all times. Both RTS and CTS packets are sent omnidirectionally. Hence, a transmitter does not need to know the location of the intended receiver. As compared to 802.11, the silenced region is not reduced by the proposed protocol, spatial reuse is thus not increased. The authors observe that the mobility of nodes does not affect throughput. However, they note that their mobility model only allows for position changes at discrete intervals of time.

In the protocol of Sanchez et al. [Sánchez et al., 2001], CTS and DATA packets are transmitted using directional antennas. The authors argue that an omnidirectional transmission of RTS packets reduces the hidden terminal problem, whereas a directional transmission reduces the exposed terminal problem. The authors propose two corresponding schemes of their protocol. By means of simulation, they conclude that a directional transmission of RTS packets generally outperforms the omnidirectional scheme. The authors further elaborate on the performance impact of the carrier sensing threshold. They conclude that without carrier detection the hidden terminal problem dominates over the exposed terminal problem.

The Directional Virtual Carrier Sensing (DVCS) approach [Takai et al., 2002] deploys a directional RTS/CTS exchange and a timer-controlled DNAV table. Location information is cached whenever a node overhears signals. This information is used when setting up a communication. If a node does not have up-to-date location information about its intended receiver in its cache, or if no CTS packet was received upon directional transmission of a RTS packet, the RTS packet is sent omnidirectionally. The authors refer to this mechanism as angle of arrival (AOA) caching. Power control is assumed in order to not extend the transmission range beyond the omnidirectional range. This work is one of the few studies where mobility is considered in the simulations. The authors report that in the case of mobility, physical carrier sensing brings about a dramatic performance improvement, in particular if nodes experience an accumulated interference due to numerous concurrent transmissions. In such cases, where nodes may fail to receive control packets (RTS or CTS) successfully, physical carrier sensing can effectively help to avoiding collisions.

The MAC protocol of Lal et al. [Lal et al., 2002] is receiver-based and allows for simultaneous receptions (receiver-SDMA). A particular aim is to increase the throughput at nodes that lie on many active nodes, so called bottleneck nodes. A receiver synchronizes a number of packet receptions from other nodes. This is done by polling neighboring nodes by means of a periodically transmitted ready-to-receive (RTR) packet. The following RTS/CTS 
handshake (RTS packets contain training sequences for the purpose of beamforming) and data transmission is fully directional.

The Dual Busy Tone Multiple Access with Directional Antennas (DBTMA/DA) protocol [Huang et al., 2002] adapts the Dual Busy Tone Multiple Access (DBTMA) protocol to directional antennas. The idea here is to transmit tones directionally in addition to the directional transmission of control packets. This is motivated by the authors' observation that the performance of MAC protocols relying on the RTS/CTS scheme deteriorates in cases of control packet collisions. With DBTMA/DA, a 'transmit busy tone' and 'receive busy tone' is transmitted along with data transmission and reception, respectively. This provides a means for alleviating the hidden and exposed terminal problem. Numerical work showed remarkable beneficial effects on throughput and end-to-end delay. The authors also investigate the omnidirectional use of busy tones as well as hybrid schemes. The directional transmission of transmit busy tones turns out to be superior over the omnidirectional transmission, since it helps to avoid the exposed terminal problem. The performance comparison of directional and omnidirectional receive busy tones is less straight forward. In fact, a tradeoff exists. Omnidirectional receive busy tones do not result in new hidden terminal problems, but reduce spatial reuse. In contrast, directional receive busy tones provide better spatial reuse, but suffer from new hidden terminal problems. It should be noted that, by using busy tones, the maintenance of DNAV tables can be avoided.

A MAC protocol for full exploitation of directional antennas is proposed by Korakis et al. in [Korakis et al., 2003]. The most distinct protocol aspect is the rotational directional transmission of RTS packets. This ensures a directional communication setup without requiring knowledge about the receiver location. However, multiple RTS packets transmitted for a single data packet may degrade the MAC performance. Note that another MAC-mechanism using rotational beamforming was proposed by Roy et al. [Roy et al., 2003]. Another major aspect is the use of a location table, maintaining the identity of each detected neighbor, the beam index on which it can be reached, and the corresponding beam index used by the neighbor. The location table is updated upon each packet reception and keeps track of the angular direction of neighboring nodes. Second, it is used for blocking beam directions that are skipped in the circular RTS transmission. The method seems to be particularly vulnerable to antenna rotation. The maintenance of location tables induces overhead and may not be suitable for highly dynamic scenarios.

The Smart-802.11 b protocol [Singh and Singh, 2004] is based on 802.11 and deploys beamforming, a direction-of-arrival algorithm and nulling. A transmitter must transmit a sender-tone and must not transmit the data packet before receiving a receiver-tone. The sender- and receiver-tones serve as a substitute for the conventional RTS/CTS exchange. Both tones are transmitted 
directionally. The sender-tone is used for beamforming at the receiver, i.e. both beamforming toward the transmitter and nulling toward interferers. A receiving node estimates the direction of the transmitter as the direction with the maximum received signal strength. As compared to RTS packets, no destination node can be indicated by the sender-tone. Thus, a receiver may receive a data packet that was not intended for it. Note that tones preceding control packet transmissions are also used in reference [Roy et al., 2003].

\section{2 "Non-802.11"-type MAC protocols for directional antennas}

The Receiver-Oriented Multiple Access (ROMA) protocol [Bao and GarciaLuna-Aceves, 2002] is the only scheduled MAC protocol discussed in this survey. Relying on local two-hop topology information, ROMA splits nodes into transmitters and receivers, which are paired together for maximum throughput. This allows for transmission-SDMA and reception-SDMA. The separation into transmitters and receivers is carried out in a random fashion. The ROMA protocol comprises the steps of priority-assignment, transmission/reception mode assignment, hidden terminal avoidance and selection of simultaneous receivers (for transmitting nodes) and simultaneous transmitters (for receiving nodes). In this survey, ROMA is the only protocol having explicit means for supporting QoS. A weight associated with each link reflects the data flow demand governed by upper layers of the transmitter and is used for contention resolution.

The Direction-of-arrival MAC (DOA-MAC) protocol [Singh and Singh, 2003 ] is a time-slotted approach based on the Slotted-ALOHA protocol. With DOA-MAC, time slots are broken into three mini slots. During the first slot, each transmitter transmits a tone toward the intended receiver. The receiving nodes run a direction-of-arrival algorithm and lock their beam toward the strongest signal. Furthermore, the receivers form nulls toward all the other identified directions. Data packets are transmitted during the second mini slot. A packet is not acknowledged in the third mini slot if the receiving node was not the intended receiver. False packet receptions may occur since no RTS/CTS handshake is performed before data transmission. This is why this approach is considered to allow for a high amount of randomness. Besides throughput, additional performance measures would be interesting for the evaluation of DOA-MAC, in particular the probability of unintended packet receptions and the probability of deadlocks. The authors proposed a similar approach [Singh and Singh, 2004], called Smart-Aloha. As an enhancement of DOA-MAC the authors implemented a single-entry cache scheme. It allows a receiver to beamform toward the second strongest signal if the receiver was not the intended receiver of a packet transmitted by a node providing the strongest signal. 
Table 1. Protocol comparison: Usage of omnidirectional (o), directional (d) and rotational directional (rot) antenna patterns

\begin{tabular}{lccccccccccccc}
\hline \multicolumn{1}{c}{$\begin{array}{c}\text { phys. } \\
\text { carrier RTR }\end{array}$} & RTS & CTS & DATA & ACK & tones \\
& sensingTXRX TX RXTXRXTX RX TXRX & t/r \\
\hline IEEE 802.11 DCF & $\mathrm{o}$ & - & - & $\mathrm{o}$ & $\mathrm{o}$ & $\mathrm{o}$ & $\mathrm{o}$ & $\mathrm{o}$ & $\mathrm{o}$ & $\mathrm{o}$ & $\mathrm{o}$ & - \\
\hline D-MAC scheme 1 & $\mathrm{d}$ & - & - & $\mathrm{d}$ & $\mathrm{o}$ & $\mathrm{o}$ & $\mathrm{o}$ & $\mathrm{d}$ & $\mathrm{o}$ & $\mathrm{d}$ & $\mathrm{o}$ & - \\
D-MAC scheme 2 & $\mathrm{d}$ & - & - & $\mathrm{o} / \mathrm{d}$ & $\mathrm{o}$ & $\mathrm{o}$ & $\mathrm{o}$ & $\mathrm{d}$ & $\mathrm{o}$ & $\mathrm{d}$ & $\mathrm{o}$ & - \\
MMAC & $\mathrm{d}$ & - & - & $\mathrm{d}$ & $\mathrm{o}$ & $\mathrm{d}$ & $\mathrm{d}$ & $\mathrm{d}$ & $\mathrm{d}$ & $\mathrm{d}$ & $\mathrm{d}$ & - \\
ToneDMAC & $\mathrm{d}, \mathrm{o}$ & - & - & $\mathrm{d}$ & $\mathrm{o}$ & $\mathrm{d}$ & $\mathrm{o}$ & $\mathrm{d}$ & $\mathrm{o}$ & $\mathrm{d}$ & $\mathrm{o}$ & $\mathrm{o} / \mathrm{o}$ \\
Nasipuri et al. & $\mathrm{o}$ & - & - & $\mathrm{o}$ & $\mathrm{o}$ & $\mathrm{o}$ & $\mathrm{o}$ & $\mathrm{d}$ & $\mathrm{d}$ & - & - & - \\
Sanchez et al. & $\mathrm{o}$ & - & $-\mathrm{d}(\mathrm{o})$ & $\mathrm{o}$ & $\mathrm{d}$ & $\mathrm{d}$ & $\mathrm{d}$ & $\mathrm{d}$ & - & - & - \\
DVCS & $\mathrm{o} / \mathrm{d}$ & - & - & $\mathrm{d} / \mathrm{o}$ & $\mathrm{d}$ & $\mathrm{d}$ & $\mathrm{d}$ & $\mathrm{d}$ & $\mathrm{d}$ & $\mathrm{d}$ & $\mathrm{d}$ & - \\
Lal et al. & & $\mathrm{o} / \mathrm{d}$ & $\mathrm{o}$ & $\mathrm{d}$ & $\mathrm{d}$ & $\mathrm{d}$ & $\mathrm{d}$ & $\mathrm{d}$ & $\mathrm{d}$ & $\mathrm{d}$ & $\mathrm{d}$ & - \\
DBTMA/DA & $\mathrm{o}$ & - & - & $\mathrm{o}$ & & $\mathrm{d}$ & & $\mathrm{d}$ & & - & - & $\mathrm{d}(\mathrm{o}) / \mathrm{d}(\mathrm{o})$ \\
Korakis et al. & $\mathrm{o}$ & - & - & $\mathrm{rot}$ & $\mathrm{o}$ & $\mathrm{d}$ & $\mathrm{o}$ & $\mathrm{d}$ & $\mathrm{d}$ & $\mathrm{d}$ & & - \\
Smart-802.11 b & - & - & - & - & - & - & - & $\mathrm{d}$ & $\mathrm{d}$ & $\mathrm{d}$ & $\mathrm{d}$ & $\mathrm{d} / \mathrm{d}$ \\
\hline ROMA & - & - & - & - & - & - & - & $\mathrm{d}$ & $\mathrm{d}$ & - & - & - \\
DOA-MACl & - & - & - & - & - & - & - & $\mathrm{d}$ & $\mathrm{d}$ & $\mathrm{d}$ & $\mathrm{d}$ & $\mathrm{d} /-$ \\
Smart-Aloha & & & & & & & & & \\
\hline
\end{tabular}

Table 2. Protocol comparison: Antenna model and assumptions

\begin{tabular}{|c|c|c|c|c|c|c|c|}
\hline \multirow[b]{2}{*}{ D-MAC } & \multicolumn{2}{|c|}{$\begin{array}{l}\text { side lobe } \\
\text { antenna (interference) } \\
\text { type model }\end{array}$} & \multicolumn{2}{|c|}{$\begin{array}{l}\text { exploitsdirect. } \\
\text { adap. range }\end{array}$} & DO & \multicolumn{2}{|r|}{$\begin{array}{l}\text { static } \\
\text { antenna } \\
\text { orient. }\end{array}$} \\
\hline & switched & omnidirect. & $\mathrm{n} / \mathrm{a}$ & - & - & - & - \\
\hline MMAC & steered & flat-top & $\mathrm{n} / \mathrm{a}$ & yes & yes & yes & - \\
\hline ToneDMAC & switched & sidelobes & $\mathrm{n} / \mathrm{a}$ & yes & yes & - & - \\
\hline Nasipuri et al. & switched & ideal sect. & $\mathrm{n} / \mathrm{a}$ & - & - & - & yes \\
\hline Sanchez et al. & switched & flat-top & $\mathrm{n} / \mathrm{a}$ & - & - & - & - \\
\hline DVCS & adaptive & realistic & - & - & - & -- & - \\
\hline Lal et al. & adaptive & realistic & yes & - & - & - & yes \\
\hline DBTMA/DA & switched & omnidirect. & $\mathrm{n} / \mathrm{a}$ & - & - & - & - \\
\hline Korakis et al. & switched & ideal sect. & $\mathrm{n} / \mathrm{a}$ & yes & yes & - & yes \\
\hline Smart-802.11b & badaptive & realistic & yes & yes & yes & - & - \\
\hline ROMA & adaptive & no side lob. & ideal & - & - & - & - \\
\hline $\begin{array}{l}\text { DOA-MAC/ } \\
\text { Smart-Aloha }\end{array}$ & adaptive & realistic & yes & yes & yes & - & - \\
\hline
\end{tabular}


Table 3. Protocol comparison: Classification, node coordination, mobility

\begin{tabular}{|c|c|c|c|c|c|c|c|c|}
\hline & $\begin{array}{l}\text { sched- } \\
\text { uled }\end{array}$ & $\begin{array}{l}\text { re- } \\
\text { ceiver } \\
\text { - initi- } \\
\text { ated }\end{array}$ & $\begin{array}{l}\text { requires } \\
\text { rreceiver } \\
\text { location } \\
\text { inform. }\end{array}$ & $\begin{array}{l}\text { nuns } \\
\text { DOA- } \\
\text { algo- } \\
\text { rithm }\end{array}$ & $\begin{array}{r}{ }^{\circ} \\
\text { TX/RX b } \\
\text { SDMA }\end{array}$ & $\begin{array}{l}\text { amount } \\
\text { of neigh- } \\
\text { borhood } \\
\text { inform. }\end{array}$ & $\begin{array}{c}\text { ran- } \\
\text { dom- } \\
\text { ness }\end{array}$ & $\begin{array}{l}\text { mobil- } \\
\text { ity in } \\
\text { simu- } \\
\text { lations }\end{array}$ \\
\hline D-MAC & - & - & yes & - & $-1-$ & low & - & - \\
\hline MMAC & - & - & yes, DD & yes & $-1-\mathrm{n}$ & medium & - & - \\
\hline ToneDMAC & - & - & yes & select & $-1-$ & low & - & - \\
\hline Nasipuri et al. & - & - & - & select & $-1-$ & low & - & yes \\
\hline Sanchez et al. & - & - & yes & select & $-1-$ & low & - & - \\
\hline DVCS & - & - & yes & yes & $-1-\mathrm{n}$ & medium & - & yes \\
\hline Lal et al. & - & yes & - & yes & - yes $n$ & medium & some & - \\
\hline DBTMA/DA & - & - & - & select & $-1-$ & low & - & - \\
\hline Korakis et al. & - & - & - & select & $-1-$ & high & - & - \\
\hline Smart- $802.11 \mathrm{~b}$ & - & - & yes & yes & $-1-n$ & medium & nedium & - \\
\hline ROMA & yes & yes & & yes & yes/yes & high & some & - \\
\hline $\begin{array}{l}\text { DOA-MAC/ } \\
\text { Smart-Aloha }\end{array}$ & - & - & yes & yes & $-i-n$ & medium & high & - \\
\hline
\end{tabular}

\section{Comparison of MAC Protocols for Directional Antennas}

Tables 1-3 summarize and compare the main properties of the discussed MAC protocols. In case of absent information, entries were left blank. Table 1 indicates which message type is sent using omnidirectional (o) or directional (d) antenna mode; the usage of tones, which may serve different purposes, is indicated in the last column. Table 2 lists the used antenna type, along with the modeling assumptions of the respective papers. It shows how the authors model side lobes, whether they exploit nulling or range extension and whether they assume a static antenna orientation.

Table 3 further categorizes the protocols and summarizes the required information from and coordination with neighboring nodes. The amount of neighborhood information is considered to be low if nodes simply keep track of RTS and CTS packets. Maintaining a DNAV table or gathering information for adaptive nulling gives raise to a medium amount of neighborhood information, whereas any further need for neighborhood information is regarded as resulting in a high amount. Table 3 further indicates whether mobility is considered by the authors.

There may be different reasons why certain protocols consciously allow for randomness, such as "hit-or-miss" transmissions or a relaxed protection of transmission against interference. Due to the dynamics in mobile systems, 
the lack of network infrastructure in ad hoc networks, and the peculiarities of using directional antennas, a certain degree of randomness may be unavoidable or even beneficial for the network performance by reducing protocol overhead.

We also observe that many of the discussed protocols employ the idea of a directional network allocation vector (DNAV) table. While a conventional network allocation vector (NAV) indicates the resource allocation-detected e.g. by the reception of a RTS or CTS packet-irrespective of the angle of arrival, the DNAV table keeps track of the resource allocation in a directionspecific manner. To this end, the elements of the DNAV table typically represent a quantized angle of arrival. Different policies are conceivable for setting the DNAV table. In the simplest case, those directions are set, from which RTS or CTS packets were received. If a node has detailed information about its surrounding topology and ongoing transmissions, more complex policies may be considered. The use of DNAV tables adds complexity, but is helpful in managing spatial reuse. If control packets include the duration of the following communication, the problem of outdated DNAVs can be avoided.

\section{Conclusions}

The application of directional antennas in ad hoc networks is expected to increase the system performance but requires appropriate MAC protocols. These should exploit the benefits of directional transmission, i.e., reduce interference, increase the link quality, and increase the spatial reuse. Although directional transmission and/or reception reduces the reserved transmission floor, a fundamental tradeoff between spatial reuse and packet collisions generally resides in the surveyed protocols.

This paper gave a survey and classification of MAC protocols in this area. Most of the protocols are extensions to IEEE 802.11, using different combinations of directional and omnidirectional messages. While most of them seem to increase the performance compared to standard 802.11, we believe that further work in this area is needed to exploit the benefits of beamforming to a further extend. From our point of view, taking an approach that goes away from the RTS/CTS paradigm of 802.11, and in particular an approach that uses outband signaling, seems to be an interesting option.

\section{References}

Bao, L. and Garcia-Luna-Aceves, J.J. (2002). Transmission scheduling in ad hoc networks with directional antennas. In Proc. ACM MobiCom.

Choudhury, R. R. and Vaidya, N. H. (2003). Deafness: A mac problem in ad hoc networks when using directional antennas. Technical report, University of Illinois at Urbana-Champaign.

Choudhury, R. R., Yang, X., Vaidya, N. H., and Ramanathan, R. (2002). Using directional antennas for medium access control in ad hoc networks. In Proc. ACM MobiCom. 
Godara, L. C. (1997). Application of antenna arrays to mobile communications, part II: Beamforming and direction-of-arrival considerations. Proceedings of the IEEE, 85(8):1195-1245.

Gummalla, A. C. V. and Limb, J. O. (2000). Wireless medium access control protocols. IEEE Commun. Surveys and Tutorials, 3(2).

Gupta, P. and Kumar, P. R. (2000). The capacity of wireless networks. IEEE Trans. Inform. Theory, 46(2):388-404.

Huang, Z., Shen, C.-C., Srisathapornphat, C., and Jaikaeo, C. (2002). A busy-tone based directional MAC protocol for ad hoc networks. In Proc. IEEE Milcom.

Ko, Y.-B., Shankarkumar, V., and Vaidya, N. H. (2000). Medium access control protocols using directional antennas in ad hoc networks. In Proc. IEEE Infocom.

Korakis, T., Jakllari, G., and Tassiulas, L. (2003). A MAC protocol for full exploitation of directional antennas in ad-hoc wireless networks. In Proc. ACM MobiHoc.

Lal, D., Toshniwal, R., Radhakrishnan, R., Agrawal, D., and Caffery, J. (2002). A novel MAC layer protocol for space division multiple access in wireless ad hoc networks. In Proc. IC $3 N$.

Nasipuri, A., Ye, S., and Hiromoto, R. E. (2000). A mac protocol for mobile ad hoc networks using directional antennas. In Proc. IEEE WCNC.

Ramanathan, R. (2001). On the performance of ad hoc networks with beamforming antennas. In Proc. ACM MobiHoc.

Roy, S., Saha, D., Bandyopadhyay, S., Ueda, T., and Tanaka, S. (2003). A network-aware MAC and routing protocol for effective load balancing in ad hoc wireless networks with directional antenna. In Proc. ACM MobiHoc.

Singh, H. and Singh, S. (2003). A MAC protocol based on adaptive beamforming for ad hoc networks. In Proc. IEEE PIMRC.

Singh, H. and Singh, S. (2004). Smart-802.1 lb MAC protocol for use with smart antennas. In Proc. IEEE ICC.

Sánchez, M., Giles, T., and Zander, J. (2001). CSMA/CA with beam forming antennas in multihop packet radio. In Proc. Swedish Workshop on Wireless Ad hoc Networks.

Takai, M., Martin, J., Ren, A., and Bagrodia, R. (2002). Directional virtual carrier sensing for directional antennas in mobile ad hoc networks. In Proc. ACM MobiHoc. 